\title{
Risk factors for lymph node metastasis in non-sentinel node basins in early gastric cancer: sentinel node concept
}

\author{
Masashi Takeuchi ${ }^{1} \cdot$ Hiroya Takeuchi $^{1,2} \cdot$ Hirofumi Kawakubo $^{1} \cdot$ Ayako Shimada ${ }^{1} \cdot$ Tadaki Nakahara $^{3} \cdot$ \\ Shuhei Mayanagi ${ }^{1} \cdot$ Masahiro Niihara ${ }^{4} \cdot$ Kazumasa Fukuda $^{1} \cdot$ Rieko Nakamura $^{1} \cdot$ Koichi Suda $^{1} \cdot$ Norihito Wada $^{1}$. \\ Yuko Kitagawa'
}

Received: 15 March 2018 / Accepted: 15 May 2018 / Published online: 24 May 2018

(c) The International Gastric Cancer Association and The Japanese Gastric Cancer Association 2018

\begin{abstract}
Background Sentinel node (SN) concept is being applied to early gastric cancer. However, when SNs are positive for metastasis, it is unclear how often LNs in other LN basins show metastasis. We aimed to investigate LN metastasis possibility in LN basins without SNs (non-SN basins). We determined risk factors for metastasis in non-SN basins and identified a prediction model for non-SN basin metastasis using classification and regression tree (CART) analysis.

Methods We enrolled 550 patients who were diagnosed with cT1N0M0 or cT2N0M0 gastric cancer with a single lesion and underwent SN mapping. We adopted a dual-tracer method using a radioactive colloid and blue dye to detect SNs.

Results Of all, 45 (8.2\%) patients had SN metastasis; we divided them into two groups: LN metastasis positive and $\mathrm{LN}$ metastasis negative in non-SN basins. Univariate analysis showed that the groups differed significantly regarding lymphatic invasion $(p=0.007)$, number of identified SNs $(p=0.032)$, and macrometastasis in SN basins $(p=0.005)$. The CART decision tree for predicting LN metastasis in non-SN basins had area under the curve value of 0.86 . Moreover, there were significantly differences in cancer-specific survival (CSS) between the two groups $(p=0.028)$.

Conclusions Macrometastasis in SN basins, lymphatic invasion, and number of identified SNs $\geq 5$ are risk factors for LN metastasis in non-SN basins among gastric cancer patients. We identified a prediction model with CART analysis; patients with macrometastasis in SN basins and lymphatic invasion were considered to be at the highest risk for LN metastasis.
\end{abstract}

Keywords Stomach neoplasms · Gastrectomy · Sentinel lymph node biopsy

Hiroya Takeuchi

htakeuchi@a6.keio.jp

1 Department of Surgery, Keio University School of Medicine, 35 Shinanomachi, Shinjuku-ku, Tokyo 160-8582, Japan

2 Department of Surgery, Hamamatsu University School of Medicine, 1-20-1 Handayama, Higashi-ku, Hamamatsu, Shizuoka 431-3192, Japan

3 Department of Radiology, Keio University School of Medicine, 35 Shinanomachi, Shinjuku-ku, Tokyo 160-8582, Japan

4 Division of Esophageal Surgery, Shizuoka Cancer Center Hospital, 1007 Shimonagakubo, Nagaizumi-cho, Sunto-gun, Shizuoka 411-8777, Japan

\section{Introduction}

In Japan, owing to the improvement in screening programs, the detection of localized cancer is more frequent than before, and pathological $\mathrm{T} 1$ cancer has been reported to account for $51.2 \%$ of all cancers in this country [1,2]. The possibility of lymph node (LN) metastasis is low in early gastric cancer; however, distal or total gastrectomy with D1 or D2 lymphadenectomy, which is performed for advanced cancer, is mainly selected for treating early gastric cancer [3]. Therefore, our previous studies investigated the establishment of individualized, minimally invasive treatments using sentinel node ( $\mathrm{SN}$ ) concept for early gastric cancer $[3,4]$.

The SN concept is being applied to early gastric cancer, same as melanoma [5, 6] and breast cancer [7]. According to the $\mathrm{SN}$ concept, if $\mathrm{SNs}$ are negative for metastasis, LNs are considered to not have any possibility of metastasis. 
LNs are divided into five $\mathrm{SN}$ basins along with five main gastric arteries as follows: left gastric artery (1-GA) basin, right gastric artery (r-GA) basin, right gastroepiploic artery (r-GEA) basin, left gastroepiploic artery (l-GEA) basin, and posterior gastric artery (p-GA) basin. This could help prevent unnecessary prophylactic regional $\mathrm{LN}$ dissection. The feasibility of SN mapping according to the SN concept has been reported by some institutions, including our institution $[3,4,8,9]$. Moreover, gastrectomy with SN mapping, called SN navigation surgery, could positively impact both early phase recovery and late-phase function, such as the prevention of dumping syndrome or weight loss, along with function-preserving surgeries, including partial gastrectomy, pylorus-preserving distal gastrectomy, segmental gastrectomy, and proximal gastrectomy if all $\mathrm{SNs}$ are negative for metastasis [4, 10-14].

However, when SNs are positive for metastasis, it is unclear how often LNs in other LN basins show metastasis. Therefore, D2 gastrectomy is required to be performed for patients with SN positivity [15].

We hypothesized that some patients do not need to undergo D1 or D2 gastrectomy but only need resection of the $\mathrm{SN}$ basins, even if SNs are positive for metastasis. The aim of this study was to investigate the possibility of LN metastasis in LN basins without SNs (non-SN basins) and to analyze the distribution of $\mathrm{LN}$ metastasis in patients with $\mathrm{SN}$ metastasis. Moreover, we aimed to determine the risk factors for metastasis in non-SN basins and to identify a prediction model for non-SN basin metastasis using classification and regression tree (CART) analysis.

\section{Materials and methods}

\section{Patients}

We enrolled 550 patients who were diagnosed with cT1N0M0 or cT2N0M0 gastric cancer with a single lesion and underwent gastric surgery with SN mapping as the primary treatment for gastric cancer at the Keio University, Tokyo, Japan, between November 1999 and September 2016.

Before treatment, patients were examined using upper gastrointestinal endoscopy, thoracic, and abdominal computed tomography, laboratory tests, and gastrography. Gastric cancer was diagnosed on the basis of pathological findings. In addition, the clinical cancer stage was determined according to the Japanese gastric cancer treatment guidelines (4th edition) [16] and the TNM classification [17]. We excluded patients who had previously undergone endoscopic mucosal resection (EMR)/endoscopic submucosal dissection (ESD) for the same lesion, because the validity and accuracy of SN biopsy after endoscopic treatment have not been fully investigated [18]. Moreover, we excluded patients who had undergone wedge resection, segmental resection, and pylorus-preserving gastrectomy. Overall survival (OS) was calculated from the primary treatment date. Cancer-specific survival (CSS) was also indicated the term from treatment date to the date of death from the cancer.

\section{SN mapping and surgical procedures}

We adopted a dual-tracer method involving a radioactive colloid and blue dye to detect SNs [4, 19]. We endoscopically injected a $2.0 \mathrm{ml}$ solution of technetium- $99 \mathrm{~m}$ tin colloid (150 MBq) into four quadrants of the submucosal layer surrounding the primary tumor the day before surgery. In addition, we intraoperatively injected blue dye (indocyanine green or $1 \%$ isosulfan blue) in the same manner. Within 15 min of injecting the dye, we could detect LNs and lymphatic vessels, which visually stained blue [4]. Simultaneously, a hand-held gamma probe (GPS navigator; RMD Instruments, Watertown, MA, USA) was used to detect radioactive SNs. We diagnosed LNs with radioactivity $>10$ times the background activity and/or LNs that stained blue as SNs. As mentioned above, the gastric lymphatic compartment was divided into the following five areas: 1-GA basin, which includes LNs 1, 3a, and 7; r-GA basin, which includes LNs 3b, 5, and 8a; 1-GEA basin, which includes LNs 4sa and 4sb; r-GEA basin, which includes LNs 4d and 6; and p-GA basin, which includes LN 11p [20]. Moreover, we considered the other LNs surrounding the stomach (LNs 2, $9,10,11 \mathrm{~d}$, and 14v) as a sub-basin. According to the TNM classification system, a metastasis $<0.2 \mathrm{~cm}$ was regarded as micrometastasis (MM), and single tumor cells or small clusters of cells measuring no more than $0.2 \mathrm{~mm}$ in the greatest extent, which could be detected using routine hematoxylin and eosin (HE) staining, were assessed [17, 21-24].

SN metastasis was diagnosed by intraoperative histologic examination with HE staining using a cut surface of a frozen section of each SN [25]. According to the locations of the main tumor and SN basin and their sizes, we performed total, distal, or proximal gastrectomy. This study was approved by the institutional review board of the Keio University School of Medicine, and informed consent for the entire procedure of SN mapping was obtained from all the patients before surgery.

\section{Statistical analysis}

We used $X^{2}$ test for univariate analysis to analyze categorical variables and Mann-Whitney $U$ test to analyze continuous variables. Moreover, we used CART analysis to identify the prediction model for non-SN basin metastasis using R, version 3.1.2 (R Foundation Statistical Computing, Vienna, Austria). CART analysis is a machine-learning method for 
constructing prediction models, and it simulates the clinical decision process $[26,27]$. It uses a generalization of the binomial variance called the Gini index, and there are some advantages with its use, including quick prediction and easy visual recognition of important variables [27]. We started with a single node and then assessed binary distinctions that provide most information about the class until a stop criterion was satisfied [28].

The accuracy of the results of CART analysis for the prediction of non-SN basin metastasis was confirmed using the area under the curve (AUC) determined from the receiver operator characteristics curve analysis. The association between LN metastasis in non-SN basins and long-term prognosis was assessed using the Kaplan-Meier method and log-rank tests. The R package "rpart" was used [27]. Statistical analyses were performed using Stata/SE 12.1 for Mac (Stata Corp, College Station, TX, USA). $p$ value $<0.05$ was considered significant.

\section{Results}

\section{Background characteristics}

Of all the patients, 45 (8.2\%) had SN metastasis; they included 27 men and 18 women (Table 1). The mean patient age was $62.7 \pm 12.4$ years (median age, 61 years). Clinicopathological characteristics of the patients are shown in Table 1. The main tumor location was the middle gastric body (upper, 5; middle, 23; and lower, 17). Clinical T1 (91\%) was the most frequent clinical classification before treatment, and pathological T1 (78\%) was the most frequent classification after treatment. Of the 45 patients, 39 (87\%) underwent distal gastrectomy, 2 (4\%) underwent total gastrectomy, and 4 (9\%) underwent proximal gastrectomy.

\section{SN mapping}

The number of identified SNs was 5.2 \pm 3.1 , whereas the number of metastatic SNs was $2.0 \pm 1.8$ (Table 2). Among the 45 patients, 34 had 1 metastatic SN basin, 9 had 2 metastatic SN basins, 1 had 3 metastatic SN basins, and 1 had 5 metastatic SN basins. The most common location of metastatic SNs was the r-GEA basin (23 cases), followed by the 1-GA basin (15 cases), r-GA basin ( 3 cases), 1-GEA basin ( 3 cases), sub-basin ( 2 cases), and p-GA basin ( 1 case). We recognized LN metastasis in non-SN basins in 11 patients. The distribution of metastatic non-SN basins was as follows: r-GEA basin ( 6 cases), r-GA basin ( 5 cases), sub-basin basin ( 2 cases) 1-GA basin (1 case), 1-GEA basin (1 case), and p-GA basin (1 case).
Table 1 Patient characteristics

\begin{tabular}{|c|c|}
\hline Characteristic & Total $(n=45)$ \\
\hline \multicolumn{2}{|l|}{ Sex } \\
\hline Male & $27(60 \%)$ \\
\hline Female & $18(40 \%)$ \\
\hline Age (years, mean $\pm S D$ ) & $62.7 \pm 12.4$ \\
\hline \multicolumn{2}{|l|}{ Clinical diagnosis } \\
\hline cT1 & $41(91 \%)$ \\
\hline $\mathrm{cT} 2$ & $4(9 \%)$ \\
\hline \multicolumn{2}{|l|}{ Location } \\
\hline Upper third & $5(11 \%)$ \\
\hline Middle third & $23(51 \%)$ \\
\hline Lower third & $17(38 \%)$ \\
\hline \multicolumn{2}{|l|}{ Circumference } \\
\hline Lesser curvature & $15(33 \%)$ \\
\hline Greater curvature & $10(22 \%)$ \\
\hline Anterior wall & $8(18 \%)$ \\
\hline Posterior wall & $11(24 \%)$ \\
\hline All around & $1(2 \%)$ \\
\hline \multicolumn{2}{|c|}{ Tumor size $(\mathrm{mm}$, mean $\pm \mathrm{SD})$} \\
\hline Major axis & $33.4 \pm 16.3$ \\
\hline Minor axis & $24.6 \pm 12.0$ \\
\hline \multicolumn{2}{|l|}{ Surgical procedure } \\
\hline Total gastrectomy & $2(4 \%)$ \\
\hline Distal gastrectomy & $39(87 \%)$ \\
\hline Proximal gastrectomy & $4(9 \%)$ \\
\hline Laparoscopy & $28(62 \%)$ \\
\hline \multicolumn{2}{|l|}{ Pathological results } \\
\hline pT1 & $35(78 \%)$ \\
\hline pT2 & $7(16 \%)$ \\
\hline pT3 & $3(7 \%)$ \\
\hline ly & $30(66 \%)$ \\
\hline $\mathrm{v}$ & $13(29 \%)$ \\
\hline
\end{tabular}

$S D$ standard deviation, $l y$ lymphatic invasion, $v$ venous invasion

\section{Risk factors for metastasis in non-SN basins}

We divided the 45 patients into the following two groups to identify the risk factors for metastasis in non-SN basins: $\mathrm{LN}$ metastasis positive in non-SN basins and LN metastasis negative in non-SN basins. We compared the background characteristics and findings of SN mapping between the groups (Table 3 ). The groups were similar in terms of mean age (positive vs. negative: $60.4 \pm 14.8$ vs. $63.4 \pm 11.7$ years; $p=0.517$ ), clinical and pathological stages, tumor location, and tumor size; they differed significantly in terms of lymphatic invasion $(p=0.007)$, number of identified SNs $(p=0.032)$, and the macrometastasis in $\mathrm{SN}$ basins $(p=0.005)$ as shown by univariate analysis. 
Table 2 Results of SN mapping

\begin{tabular}{ll}
\hline Variable & Total $(n=45)$ \\
\hline Number of identified SNs (mean \pm SD) & $5.2 \pm 3.1(1-19)$ \\
Number of metastatic SNs (mean \pm SD) & $2.0 \pm 1.8(1-10)$ \\
Number of metastatic SN basins & \\
1 & $34(76 \%)$ \\
2 & $9(20 \%)$ \\
3 & $1(2 \%)$ \\
4 & $0(0 \%)$ \\
5 & $1(2 \%)$ \\
Location of metastatic SN basins & \\
r-GA & $3(7 \%)$ \\
r-GEA & $23(51 \%)$ \\
1-GA & $15(33 \%)$ \\
1-GEA & $3(7 \%)$ \\
p-GA & $1(2 \%)$ \\
Sub-basin & $2(4 \%)$ \\
LN metastasis in non-SN basins & $11(23 \%)$ \\
Pattern of SN metastasis & \\
Macrometastasis & $29(64 \%)$ \\
Micrometastasis/isolated tumor cell & $16(36 \%)$ \\
Location of metastatic non-SN basins & \\
r-GA & $5(11 \%)$ \\
r-GEA & $6(13 \%)$ \\
1-GA & $1(2 \%)$ \\
p-GEA & $1(2 \%)$ \\
Sub-basin & $1(2 \%)$ \\
\hline
\end{tabular}

Sub-basin includes LNs 2, 9, 10, 11d, and 14v

$S N$ sentinel node, $L N$ lymph node, $S D$ standard deviation

\section{Prediction model for $\mathrm{LN}$ metastasis in non-SN basins using CART}

We used three variables including macrometastasis in $\mathrm{SN}$ basins, lymphatic invasion, and number of identified SNs that showed significant differences in univariate analysis initially in the CART analysis for predicting LN metastasis in non-SN basins (Fig. 1).

The most important parameter was macrometastasis in SN basins. Patients who had macrometastasis in SN basins, lymphatic invasion, and number of identified $\mathrm{SNs} \geq 5$ comprised the highest risk group. The CART decision tree had an AUC value of 0.86 (Fig. 2).

\section{Long-term outcomes compared with or without LN metastasis in non-SN basins}

In all 45 patients, the 5 -year OS rate was $88.8 \%$ and CSS rate was $93.5 \%$. There were four patients $(8.9 \%)$ who had recurrences during observation term: two patients had bone metastasis, one had liver metastasis and one had lung metastasis. No patients with recurrence at local or lymph node were observed. Of all four patients, three patients had lymph node metastasis in non-SN basins (Table 4). Although no significant difference with respect to OS (5-year OS: metastasis in non-SN basins, $78.8 \%$ vs. no metastasis in non-SN basins, $93.2 \%, p=0.535$ ), there were significantly differences in CSS (5-year CSS: metastasis in non-SN basins, $78.8 \%$ vs. no metastasis in non-SN basins, $100 \%, p=0.028$ ) between two groups (Fig. 3).

\section{Discussion}

In this study, we found that macrometastasis in SN basins, lymphatic invasion, and number of identified $\mathrm{SNs} \geq 5$ were risk factors for $\mathrm{LN}$ metastasis in non-SN basins among gastric cancer patients. Furthermore, we identified a prediction model with CART analysis, and it had an AUC value of 0.86 .

Although many studies have reported risk factors for $\mathrm{LN}$ metastasis in early gastric cancer [29,30], to the best of our knowledge, this is the first report to elucidate the possibility of LN metastasis in non-SN basins. Our study had two important clinical aspects. First, we could recognize the necessity to perform additional gastric resection after minimal surgery when the results of SN mapping were false negative. False-negative SN metastasis is unavoidable using intraoperative pathological diagnosis [4]. The findings of over 100 single-institution studies showed that the sensitivity of determining LN status using SN biopsy may vary across institutions, and it was found that the $\mathrm{SN}$ detection rate was 90-100\% and the accuracy of determining the LN status was $85-100 \%[4,25]$. Our previous multicenter prospective trial showed that among 57 cases who had SN metastasis, $4(7.0 \%)$ were false negative [25]. Although studies have examined SN MM using RT-PCR [31, 32], we previously reported that we could not exclude the possibility of overlooking SN MM using HE staining alone [33]. Considering the results of our study, we could decide whether or not additional gastrectomy with D2 lymphadenectomy should be performed based on postoperative pathological findings. Second, our study might help improve new treatment approaches including a combination of EMR/ESD and SN mapping. If resection could be performed beyond the endoscopic criteria with improvement in endoscopic treatment, the indications of EMR/ESD for cT1 gastric cancer could be further expanded using SN mapping [34]. In the future, EMR/ESD combined with SN mapping surgery will have the potential to be a novel minimal surgery.

In the present study, none of the patients had LN metastasis in non-SN basins with no lymphatic invasion, and EMR/ ESD followed by only SN basin resection may be acceptable 
Table 3 Risk factors of metastasis in non-SN basins

\begin{tabular}{|c|c|c|c|}
\hline & $\begin{array}{l}\text { Non-SN basin } \\
\text { positive }(n=11)\end{array}$ & $\begin{array}{l}\text { Non-SN basin } \\
\text { negative }(n=34)\end{array}$ & $p$ value \\
\hline Sex & & & 0.671 \\
\hline Male & $5(45 \%)$ & $21(62 \%)$ & \\
\hline Female & $6(55 \%)$ & $13(38 \%)$ & \\
\hline Age (years, mean $\pm S D$ ) & $60.4 \pm 14.8$ & $63.4 \pm 11.7$ & 0.517 \\
\hline Clinical diagnosis & & & 0.978 \\
\hline cT1 & $10(91 \%)$ & $31(91 \%)$ & \\
\hline $\mathrm{cT} 2$ & $1(9 \%)$ & $3(9 \%)$ & \\
\hline Location & & & 0.140 \\
\hline Upper third & $3(27 \%)$ & $2(6 \%)$ & \\
\hline Middle third & $5(45 \%)$ & $18(53 \%)$ & \\
\hline Lower third & $3(27 \%)$ & $14(42 \%)$ & \\
\hline Circumference & & & 0.951 \\
\hline Lesser curvature & $3(27 \%)$ & $12(35 \%)$ & \\
\hline Greater curvature & $3(27 \%)$ & $7(21 \%)$ & \\
\hline Anterior wall & $2(18 \%)$ & $6(18 \%)$ & \\
\hline Posterior wall & $3(27 \%)$ & $8(24 \%)$ & \\
\hline All around & 0 & $1(3 \%)$ & \\
\hline \multicolumn{4}{|l|}{ Tumor size $(\mathrm{mm}$, mean $\pm \mathrm{SD})$} \\
\hline Major axis & $29.7 \pm 13.4$ & $34.6 \pm 17.1$ & 0.465 \\
\hline Minor axis & $20.6 \pm 6.5$ & $26.0 \pm 13.2$ & 0.279 \\
\hline Pathological results & & & 0.887 \\
\hline pT1 & $10(91 \%)$ & $27(78 \%)$ & \\
\hline pT2 & $2(18 \%)$ & $5(15 \%)$ & \\
\hline pT3 & $1(9 \%)$ & $2(6 \%)$ & \\
\hline ly & $11(100 \%)$ & $19(56 \%)$ & 0.007 \\
\hline $\mathrm{v}$ & $4(36 \%)$ & $9(27 \%)$ & 0.529 \\
\hline Pathology & & & 0.293 \\
\hline Tubular adenocarcinoma & $6(55 \%)$ & $10(29 \%)$ & \\
\hline Poorly adenocarcinoma & $5(45 \%)$ & $23(68 \%)$ & \\
\hline Other & 0 & $1(3 \%)$ & \\
\hline Macrometastasis in SN basins & $11(100 \%)$ & $18(53 \%)$ & 0.005 \\
\hline Number of $\mathrm{SN}$ metastases in $\mathrm{SN}$ basins (mean $\pm \mathrm{SD}$ ) & $3.0 \pm 2.7$ & $1.7 \pm 1.2$ & 0.103 \\
\hline Number of identified SNs in SN basins (mean $\pm \mathrm{SD}$ ) & $6.5 \pm 2.8$ & $4.8 \pm 3.2$ & 0.032 \\
\hline Number of non-SN resections (mean $\pm \mathrm{SD})$ & $32.9 \pm 13.7$ & $31.0 \pm 14.8$ & 0.526 \\
\hline
\end{tabular}

$S N$ sentinel node, $S D$ standard deviation, $l y$ lymphatic invasion, $v$ venous invasion in patients with no lymphatic invasion. Furthermore, a clinical approach to confirm the accuracy and safety of SN mapping and consequent intraoperative EMR/ESD is needed.

As mentioned above, macrometastasis in $\mathrm{SN}$ basins, lymphatic invasion, and number of identified SNs were risk factors for LN metastasis in non-SN basins. No study has investigated metastasis in non-SN basins, but studies about skip metastasis in early gastric cancer have been reported. Miwa et al. classified LN metastasis in D2 as skip metastasis and suggested that the mechanisms of skip metastasis are associated with direct lymphatic flow to the affected LNs from the tumor on SN mapping [35, 36]. The risk factors for skip metastasis have been reported in some studies. The factors related to lymphatic invasion, which were identified in our study, have been shown to be associated with skip metastasis [37]. Moreover, the number of identified SNs was a risk factor for LN metastasis in non-SN basins, because it may indicate the number of lymphatic flow paths in SN basins and the causes of LN metastasis [36]. Our previous study demonstrated that the number of identified SNs in metastatic cases was significantly higher than that in non-metastatic cases. We believe that the development of the lymphatic network and a large lymphatic flow rate may affect the number of identified $\mathrm{SNs}$ and may be associated with metastasis in non-SN basins [38]. 
Fig. 1 Prediction model for LN metastasis in non-SN basins using CART analysis. $L N$ lymph node, $S N$ sentinel node, metastasis $\mathrm{LN}$ metastasis in non-SN basins, Pt patients, MIC micrometastasis, ITC isolated tumor cell, MAC macrometastasis



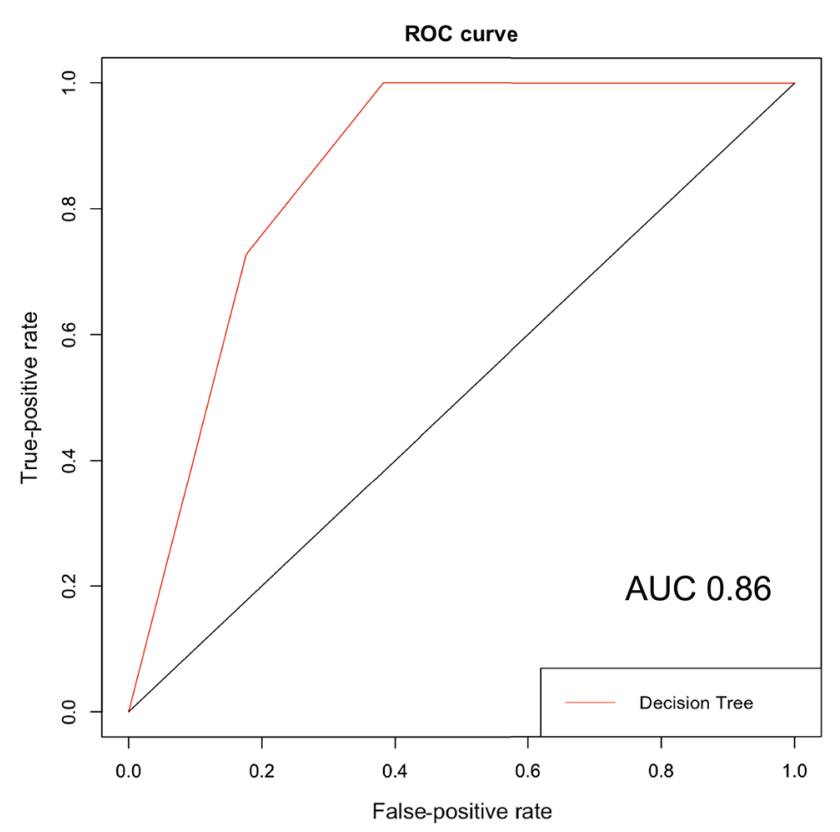

Although no significant difference with respect to OS were observed, CSS was significantly low in patients with $\mathrm{LN}$ metastasis in non-SN basins. Despite local and regional lymph node recurrences being controlled by radical D2 dissection, recurrence at distant organs were observed in patients with $\mathrm{LN}$ metastasis in non-SN basins. This suggests that they may be highly malignant than patients with no LN metastasis in non-SN basins. Therefore, our system not only helps decision-making with additional treatment, but may also lead to risk stratification of long-term prognosis. To determine more evidence for long-term outcomes, more large number of patients would be needed.

The present study has some limitations. First, this was a retrospective, single-center study limited to the Japanese population. Second, some SN basins may have been considered as non-SN basins because of the possibility of mapping error in the detection of SNs.

Fig. 2 Receiver operating characteristic curve analysis for the CART decision tree

Table 4 Recurrent patterns after gastrectomy in four patients

\begin{tabular}{lllccll}
\hline Age & Sex & $\begin{array}{l}\text { Non-SN basin } \\
\text { metastasis }\end{array}$ & $\begin{array}{l}\text { Number of SN } \\
\text { metastasis }\end{array}$ & $\begin{array}{l}\text { Number of LN } \\
\text { metastasis }\end{array}$ & $\begin{array}{l}\text { Recurrent } \\
\text { regions }\end{array}$ & $\begin{array}{l}\text { Time to recur- } \\
\text { rence (months) }\end{array}$ \\
\hline 80 & Female & Positive & 2 & 5 & Lung & 60 \\
63 & Male & Negative & 3 & 3 & Liver & 15 \\
50 & Male & Positive & 10 & 27 & Bone & 32 \\
50 & Female & Positive & 5 & 9 & Bone & 55 \\
\hline
\end{tabular}

$S N$ sentinel node, $L N$ lymph node 


\section{a Overall survival}

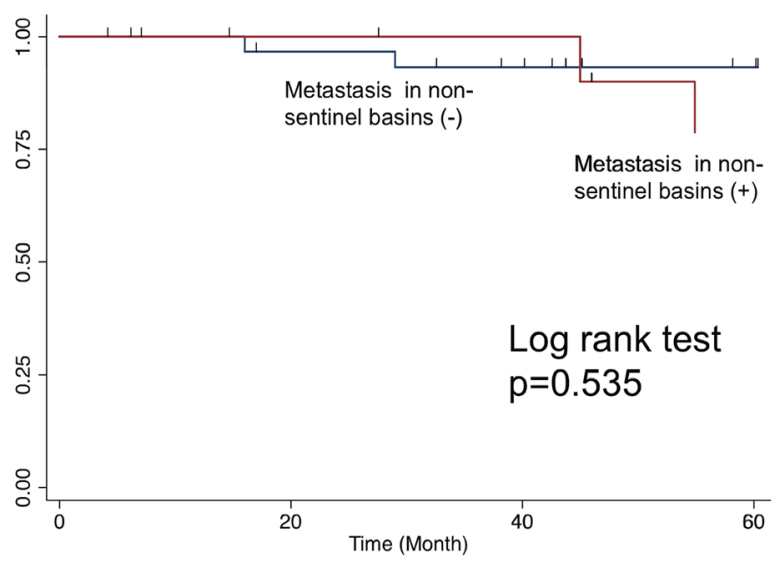

Fig. 3 Comparison of overall and cancer-specific survival outcomes for all patients with using Kaplan-Meier method. Blue lines and red lines indicated the group with lymph node metastasis in non-sentinel

\section{Conclusion}

Macrometastasis in SN basins, lymphatic invasion, and number of identified $\mathrm{SNs} \geq 5$ are risk factors for LN metastasis in non-SN basins among gastric cancer patients. A prediction model was identified with CART analysis, and patients with macrometastasis in $\mathrm{SN}$ basins and lymphatic invasion were considered to be at the highest risk. Our findings should be validated in a multicenter, prospective study in the future.

Acknowledgements The authors thank Kumiko Motooka, who is a staff member at the Department of Surgery in the Keio University School of Medicine, for her help in the preparation of this manuscript.

\section{Compliance with ethical standards}

Ethical standards All procedures were followed in accordance with the ethical standards of the responsible committee on human experimentation (institutional and national) and with Helsinki Declaration of 1964 and later versions. Informed consent or substitute for it was obtained from all patients for being included in the study.

Conflict of interest Yuko Kitagawa has received grants from Daiichi Sankyo Co., Ltd.

\section{References}

1. Isobe Y, Nashimoto A, Akazawa K, Oda I, Hayashi K, Miyashiro I, et al. Gastric cancer treatment in Japan: 2008 annual report of the JGCA nationwide registry. Gastric Cancer. 2011;14:301-16.

2. Leung WK, Wu MS, Kakugawa Y, Kim JJ, Yeoh KG, Goh KL, et al. Screening for gastric cancer in Asia: current evidence and practice. Lancet Oncol. 2008;9:279-87.

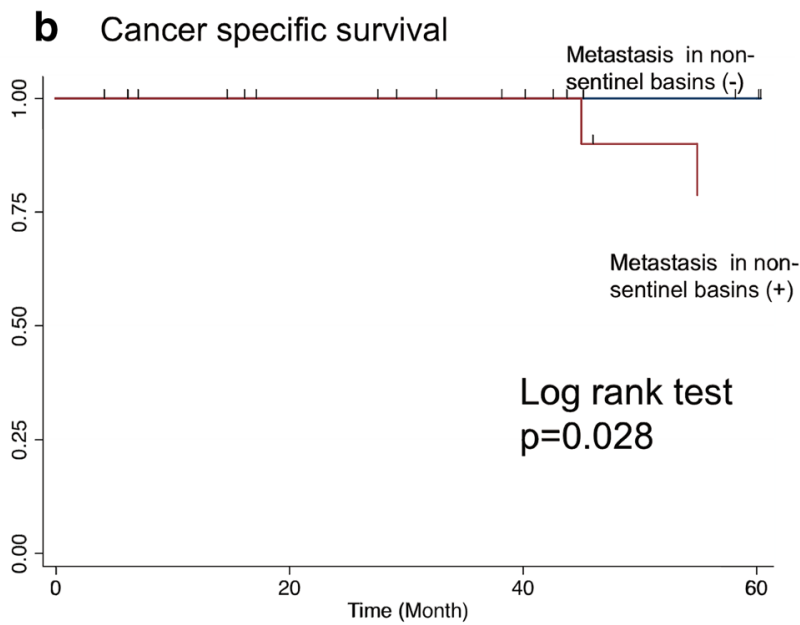

basins negative and the group with lymph node metastasis in non-SN basins positive, respectively

3. Kitagawa Y, Kitano S, Kubota T, Kumai K, Otani Y, Saikawa $\mathrm{Y}$, et al. Minimally invasive surgery for gastric cancer-toward a confluence of two major streams: a review. Gastric Cancer. 2005;8:103-10.

4. Takeuchi H, Kitagawa Y. New sentinel node mapping technologies for early gastric cancer. Ann Surg Oncol. 2013;20:522-32.

5. Wong JH, Cagle LA, Morton DL. Lymphatic drainage of skin to a sentinel lymph node in a feline model. Ann Surg. 1991;214:637-41.

6. Morton DL, Wen DR, Wong JH, Economou JS, Cagle LA, Storm FK, et al. Technical details of intraoperative lymphatic mapping for early stage melanoma. Arch Surg. 1992;127:392-9.

7. Haigh PI, Giuliano AE. Intraoperative lymphatic mapping and sentinel lymphadenectomy for breast cancer. Oper Tech Gen Surg. 2000;2:161-5.

8. Morita D, Tsuda H, Ichikura T, Kimura M, Aida S. Analysis of sentinel node involvement in gastric cancer. Clin Gastroenterol Hepatol. 2007;5:1046-52.

9. Ohdaira H, Nimura H, Mistumuro N, Takahashi N, Kashiwagi $\mathrm{H}$, Yanaga K. Validity of modified gastrectomy combined with sentinel node navigation surgery for early gastric cancer. Gastric Cancer. 2007; 10:117-22.

10. Seto Y, Nagawa H, Muto Y, Kaizaki S, Kitayama J, Muto T. Preliminary report on local resection with lymphadenectomy for early gastric cancer. Br J Surg. 1999;86:526-8.

11. Matsuda T, Kaneda K, Takamatsu M, Aishin K, Awazu M, Okamoto A, et al. Segmental gastrectomy with radical lymph node dissection for early gastric cancer. World J Gastroenterol. 2010;16:5247-51.

12. Zhang D, Shimoyama S, Kaminishi M. Feasibility of pyloruspreserving gastrectomy with a wider scope of lymphadenectomy. Arch Surg. 1998;133:993-7.

13. Takeuchi H, Goto O, Yahagi N, Kitagawa Y. Function-preserving gastrectomy based on the sentinel node concept in early gastric cancer. Gastric Cancer. 2017;20:53-9.

14. Takeuchi H, Oyama T, Kamiya S, Nakamura R, Takahashi T, Wada N, et al. Laparoscopy-assisted proximal gastrectomy with sentinel node mapping for early gastric cancer. World J Surg. 2011;35:2463-71.

15. Takeuchi H, Kitagawa Y. Sentinel lymph node biopsy in gastric cancer. Cancer J. 2015;21:21-4. 
16. Kodera Y, Sano T. Japanese gastric cancer treatment guidelines 2014 (ver. 4). Gastric Cancer. 2017;20:1-19.

17. Sobin LH, Gospodarowicz MK, Wittekind Ch, editors. TNM: classification of malignant tumours. 7th ed. Hoboken: WileyBlackwell; 2009.

18. Mayanagi S, Takeuchi H, Kamiya S, Niihara M, Nakamura R, Takahashi T, et al. Suitability of sentinel node mapping as an index of metastasis in early gastric cancer following endoscopic resection. Ann Surg Oncol. 2014;21:2987-93.

19. Shimada A, Takeuchi H, Kamiya S, Fukuda K, Nakamura R, Takahashi T, et al. Clinical significance of the anterosuperior lymph nodes along the common hepatic artery identified by sentinel node mapping in patients with gastric cancer. Gastric Cancer. 2016;19:1088-94.

20. Kinami S, Fujimura T, Ojima E, Fushida S, Ojima T, Funaki H, et al. PTD classification: proposal for a new classification of gastric cancer location based on physiological lymphatic flow. Int $\mathrm{J}$ Clin Oncol. 2008;13:320-9.

21. Nakahara T, Kitagawa Y, Takeuchi H, Fujii H, Suzuki T, Mukai $\mathrm{M}$, et al. Preoperative lymphoscintigraphy for detection of sentinel lymph node in patients with gastric cancer-initial experience. Ann Surg Oncol. 2008;15:1447-53.

22. Takeuchi H, Kitajima M, Kitagawa Y. Sentinel lymph node as a target of molecular diagnosis of lymphatic micrometastasis and local immunoresponse to malignant cells. Cancer Sci. 2008;29:441-50.

23. Yanagita $\mathrm{S}$, Natsugoe $\mathrm{S}$, Uenosono $\mathrm{Y}$, Arigami $\mathrm{T}$, Arima $\mathrm{H}$, Kozono T, et al. Detection of micrometastases in sentinel node navigation surgery for gastric cancer. Surg Oncol. 2008;17:203-10.

24. Ishii K, Kinami S, Funaki K, Fujita H, Ninomiya I, Fushida S, et al. Detection of sentinel and non-sentinel lymph node micrometastases by complete serial sectioning and immunohistochemical analysis for gastric cancer. J Exp Clin Cancer Res. 2008;27:7.

25. Kitagawa Y, Takeuchi H, Takagi Y, Natsugoe S, Terashima M, Murakami N. Sentinel node mapping for gastric cancer: a prospective multicenter trial in Japan. J Clin Oncol. 2013;31:3704-10.

26. Garzotto M, Beer TM, Hudson RG, Peters L, Hsieh YC, Barrera $\mathrm{E}$, et al. Improved detection of prostate cancer using classification and regression tree analysis. J Clin Oncol. 2005;23:4322-9.
27. Breiman L, Friedman J, Stone CJ, Olshen RA. Classification and regression trees. 1st ed. Belmont: CRC Press; 1984.

28. Williams G. Data mining with rattle and R: the art of excavating data for knowledge discovery. Use R! New York: Springer; 2011. pp. 205-244

29. Shida A, Mitsumori N, Nimura H, Takano Y, Iwasaki T, Fujisaki $\mathrm{M}$, et al. Prediction of lymph node metastasis and sentinel node navigation surgery for patients with early-stage gastric cancer. World J Gastroenterol. 2016;22:7431-9.

30. Lee K, Park DJ, Choe G, Kim HH, Kim WH, Lee HS. Increased intratumoral lymphatic vessel density correlates with lymph node metastasis in early gastric carcinoma. Ann Surg Oncol. 2010;17:73-80.

31. Osaka H, Yashiro M, Sawada T, Katsuragi K, Hirakawa K. Is a lymph node detected by the dye-guided method a true sentinel node in gastric cancer? Clin Cancer Res. 2004;10:6912-8.

32. Arigami T, Natsugoe S, Uenosono Y, Mataki Y, Ehi K, Higashi H, et al. Evaluation of sentinel node concept in gastric cancer based on lymph node micrometastasis determined by reverse transcription-polymerase chain reaction. Ann Surg. 2006;243:341-7.

33. Takeuchi H, Kitagawa Y. Sentinel node and mechanism of lymphatic metastasis. Ann Vasc Dis. 2012;5:249-57.

34. Ono H, Yao K, Fujishiro M, Oda I, Nimura S, Yahagi N, et al. Guidelines for endoscopic submucosal dissection and endoscopic mucosal resection for early gastric cancer. Dig Endosc. 2016;28:3-15.

35. Miwa K, Kinami S, Taniguchi K, Fushida S, Fujimura T, Nonomura A. Mapping sentinel nodes in patients with early-stage gastric carcinoma. Br J Surg. 2003;90:178-82.

36. Park SS, Ryu JS, Min BW, Kim WB, Kim SJ, Kim CS. Impact of skip metastasis in gastric cancer. ANZ J Surg. 2005;75:645-9.

37. Lee SE, Lee JH, Ryu KW, Cho SJ, Lee JY, Kim CG, et al. Sentinel node mapping and skip metastases in patients with early gastric cancer. Ann Surg Oncol. 2009;16:603-8.

38. Kamiya S, Takeuchi H, Nakahara T, Niihara M, Nakamura R, Takahashi T, et al. Auxiliary diagnosis of lymph node metastasis in early gastric cancer using quantitative evaluation of sentinel node radioactivity. Gastric Cancer. 2016;19:1080-7. 